\title{
Hydrides in young stellar objects: Radiation tracers in a protostar-disk-outflow system ${ }^{\star, \star \star}$
}

\author{
A. O. Benz ${ }^{1}$, S. Bruderer ${ }^{1}$, E. F. van Dishoeck ${ }^{2,3}$, P. Stäuber ${ }^{1}$, S. F. Wampfler ${ }^{1}$, M. Melchior ${ }^{1,35}$, C. Dedes $^{1}$,
} F. Wyrowski ${ }^{30}$, S. D. Doty ${ }^{19}$, F. van der Tak ${ }^{10,11}$, W. Bächtold ${ }^{36}$, A. Csillaghy ${ }^{35}$, A. Megej ${ }^{36}$, C. Monstein ${ }^{1}$, M. Soldati ${ }^{35}$, R. Bachiller ${ }^{12}$, A. Baudry ${ }^{6}$, M. Benedettini ${ }^{13}$, E. Bergin ${ }^{14}$, P. Bjerkeli ${ }^{9}$, G. A. Blake ${ }^{15}$, S. Bontemps ${ }^{6}$, J. Braine ${ }^{6}$, P. Caselli ${ }^{4,5}$, J. Cernicharo ${ }^{16}$, C. Codella ${ }^{5}$, F. Daniel ${ }^{16}$, A. M. di Giorgio ${ }^{13}$, P. Dieleman ${ }^{10}$, C. Dominik ${ }^{17,18}$, P. Encrenaz ${ }^{20}$, M. Fich ${ }^{21}$, A. Fuente ${ }^{22}$, T. Giannini ${ }^{23}$, J. R. Goicoechea ${ }^{16}$, Th. de Graauw ${ }^{10}$, F. Helmich ${ }^{10}$, G. J. Herczeg ${ }^{3}$, F. Herpin ${ }^{6}$, M. R. Hogerheijde ${ }^{2}$, T. Jacq ${ }^{5}$, W. Jellema ${ }^{10}$, D. Johnstone ${ }^{7,8}$, J. K. Jørgensen ${ }^{24}$, L. E. Kristensen ${ }^{2}$, B. Larsson ${ }^{25}$, D. Lis ${ }^{26}$, R. Liseau ${ }^{9}$, M. Marseille ${ }^{10}$, C. M ${ }^{\text {C }}$ Coey $^{21,27}$, G. Melnick ${ }^{28}$, D. Neufeld ${ }^{29}$, B. Nisini ${ }^{23}$, M. Olberg 9 , V. Ossenkopf ${ }^{34}$, B. Parise ${ }^{30}$, J. C. Pearson ${ }^{31}$, R. Plume ${ }^{32}$, C. Risacher ${ }^{10}$, J. Santiago-García ${ }^{33}$, P. Saraceno ${ }^{13}$, R. Schieder ${ }^{34}$, R. Shipman ${ }^{10}$, J. Stutzki ${ }^{34}$, M. Tafalla ${ }^{12}$, A. G. G. M. Tielens ${ }^{2}$, T. A. van Kempen ${ }^{28}$,

$$
\text { R. Visser }{ }^{2} \text {, and U. A. Yildiz }{ }^{2}
$$

(Affiliations are available on page 5 of the online edition)

Received 31 May 2010 / Accepted 14 July 2010

\section{ABSTRACT}

Context. Hydrides of the most abundant heavier elements are fundamental molecules in cosmic chemistry. Some of them trace gas irradiated by UV or X-rays.

Aims. We explore the abundances of major hydrides in W3 IRS5, a prototypical region of high-mass star formation.

Methods. W3 IRS5 was observed by HIFI on the Herschel Space Observatory with deep integration $(\simeq 2500$ s) in 8 spectral regions.

Results. The target lines including $\mathrm{CH}, \mathrm{NH}, \mathrm{H}_{3} \mathrm{O}^{+}$, and the new molecules $\mathrm{SH}^{+}, \mathrm{H}_{2} \mathrm{O}^{+}$, and $\mathrm{OH}^{+}$are detected. The $\mathrm{H}_{2} \mathrm{O}^{+}$and $\mathrm{OH}^{+} J=1-0$ lines are found mostly in absorption, but also appear to exhibit weak emission (P-Cyg-like). Emission requires high density, thus originates most likely near the protostar. This is corroborated by the absence of line shifts relative to the young stellar object (YSO). In addition, $\mathrm{H}_{2} \mathrm{O}^{+}$and $\mathrm{OH}^{+}$also contain strong absorption components at a velocity shifted relative to W3 IRS5, which are attributed to foreground clouds.

Conclusions. The molecular column densities derived from observations correlate well with the predictions of a model that assumes the main emission region is in outflow walls, heated and irradiated by protostellar UV radiation.

Key words. astrochemistry - line: identification - stars: formation - stars: massive - photon-dominated region - submillimeter: ISM

\section{Introduction}

In interstellar clouds, chemical reactions with hydrogen molecules lead to an elementary class of molecules that represent key species in the chemical evolution to larger molecules. These fundamental molecules, known as hydrides, include $\mathrm{OH}$, $\mathrm{CH}, \mathrm{NH}, \mathrm{SH}, \mathrm{H}_{2} \mathrm{O}$, and their ions, $\mathrm{OH}^{+}, \mathrm{CH}^{+}, \mathrm{NH}^{+}, \mathrm{SH}^{+}, \mathrm{H}_{2} \mathrm{O}^{+}$, and $\mathrm{H}_{3} \mathrm{O}^{+}$. The combination of hydrogen atoms with a heavier atom causes large dipole moments and large rotation constants, particularly in diatomic hydrides. This widely separates the excitation levels. Only low-J lines are excited at temperatures relevant to star and planet formation. These lines have now become observable with the Herschel Space Observatory.

Many hydrides have a high activation energy in their formation paths. However, if high-energy photons - far UV (FUV) or $\mathrm{X}$-rays - interact with the molecular gas and heat it, hydrides and particularly their ions are greatly enhanced in abundance

\footnotetext{
* Herschel is an ESA space observatory with science instruments provided by a European-led Principal Investigator consortia and with important participation from NASA.

$\star \star$ Appendix (page 5) is only available in electronic form at http://www . aanda.org
}

(e.g., Hollenbach \& Tielens 1999). Ionized hydrides are chemically even more active and can substantially drive chemical evolution. Many of the above hydrides have been observed from the ground in absorption in diffuse interstellar clouds (e.g. Swings \& Rosenfeld 1937; Menten et al. 2010; Wyrowski et al. 2010).

Here we focus on hydrides in dense star-forming regions surrounding protostars. We report first results of the "Radiation Diagnostics" project within the Herschel key program "Water In Star-forming regions with Herschel" (WISH, van Dishoeck et al., in prep.). The "Radiation Diagnostics" subprogram aims to trace high-energy radiation through the most abundant hydrides and their ions and relate them to the chemical network of water. Preparing for this project, Stäuber et al. (2004, 2005) showed that hydrides may be enhanced in star-forming regions affected by far UV and/or X-ray irradiation. In a second step, optimal lines were selected using catalogued data in the available databases or computed from measured molecular constants (Bruderer 2006).

Spherically symmetric models including FUV and X-rays have found that most hydrides would be too weak for Herschel detection. However, evidence of extended high-energy irradiation (Doty et al. 2004) and in particular the observation of $\mathrm{CO}^{+}$, 

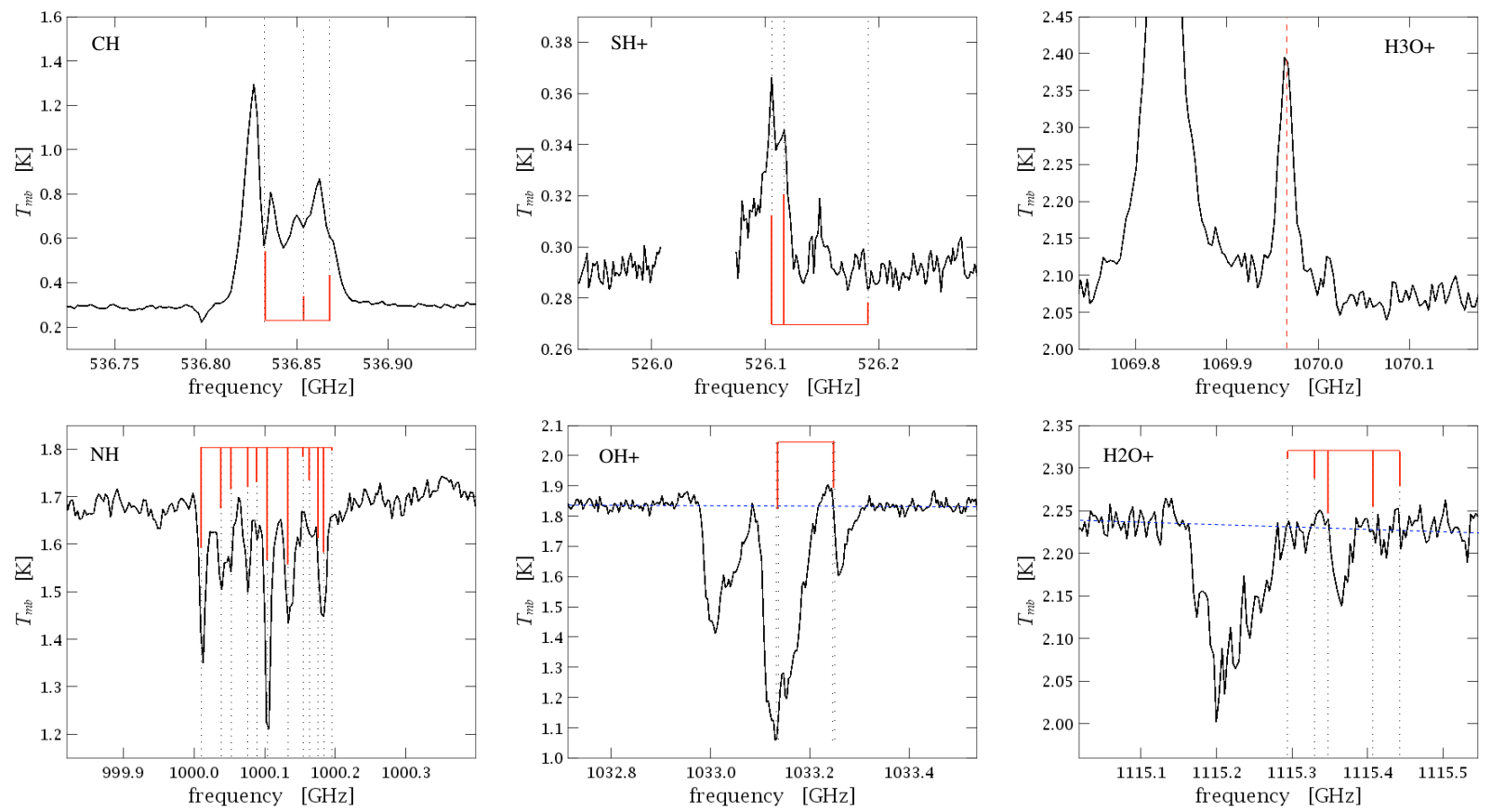

Fig. 1. Spectral lines observed towards W3 IRS5 in main beam temperature. The positions of the theoretical fine/hyperfine structure lines are shown shifted by $-38.4 \mathrm{~km} \mathrm{~s}^{-1}$, the systemic motion of the YSO. The length of the vertical bars indicates the theoretical intensities in arbitrary scale. The frequency binning is $1 \mathrm{~km} \mathrm{~s}^{-1}$.

with an abundance four orders of magnitude larger than predicted, in W3 IRS5 and other YSOs with the JCMT (Stäuber et al. 2007) raised the expectation that the effects of irradiation may be more dramatic in asymmetric reality. Bruderer et al. (2009) modeled and interpreted the $\mathrm{CO}^{+}$anomaly as FUV radiation originating in the YSOs and irradiating the walls of outflow cavities.

Based on these predictions, the "Radiation Diagnostics" observations started with deep integrations of a large number of hydrides that was to be followed by a survey of a few species in many sources of various ages and masses. Here we present exploratory observations towards W3 IRS5, a nearby region (1.83 kpc, Imai et al. 2000) of high-mass star formation, moving at $-38.4 \mathrm{~km} \mathrm{~s}^{-1}$ in the local standard of rest (LSR). W3 IRS5 resembles the Trapezium cluster in Orion but is considerably younger. At least six radio sources are in the Herschel beam. They represent "hypercompact" H II regions produced by highmass YSOs, at least two of which are O stars (van der Tak et al. 2005; Rodón et al. 2008). In this paper, we report the observations of major hydrides towards W3 IRS5 apart from $\mathrm{H}_{2} \mathrm{O}, \mathrm{CH}^{+}$, and $\mathrm{OH}$, which are studied in other projects of WISH (Chavarría et al. 2010; Wampfler et al., in prep.).

\section{Observations}

The Heterodyne Instrument for the Far Infrared (HIFI, de Graauw et al. 2010) on Herschel observed W3 IRS5 between 1 and 8 March 2010 in the Science Demonstration Phase in eight $4 \mathrm{GHz}$ frequency bands for about $2500 \mathrm{~s}$ each. One of them includes [C II] at $1900.5369 \mathrm{GHz}$. We used the wide band spectrometer which has a spectral resolution of $1.1 \mathrm{MHz}$, and HIPE 3.0 for pipeline and data analysis. The data were taken by double beam switching (DBS), the high-frequency (HEB) bands in fast DBS mode. The off-source position was at a distance of 3 arcmin in the NE and SW without remarkable IR sources.
The current accuracy of the velocity calibration is estimated to be better than $2 \mathrm{~km} \mathrm{~s}^{-1}$. The antenna temperature was converted to main beam temperature, using pre-flight antenna efficiencies. After visual inspection and defringing, the $\mathrm{V}$ polarization was shifted linearly in flux to match the $\mathrm{H}$ polarization. The two polarizations were then added. A second observation of equal length was made using a local oscillator frequency shifted by $10 \mathrm{~km} \mathrm{~s}^{-1}$. The two data sets were plotted in both upper and lower sideband presentation. If a line matched velocities in one sideband and was double with $20 \mathrm{~km} \mathrm{~s}^{-1}$ separation in the other, the frequency of the former was assumed. All lines of interest could be attributed to a sideband without ambiguity. The continuum was divided by 2 for double sideband observations, assuming that it is the same in both sidebands, of equal sensitivity.

\section{Results}

Most lines (except $\mathrm{H}_{3} \mathrm{O}^{+}$) are split by fine or hyperfine interaction as indicated in Fig. 1. Table 1 lists the observed lines (strongest only for multiples) and summarizes the quantitative observational results. Molecules here detected for the first time in star-forming regions include $\mathrm{H}_{2} \mathrm{O}^{+}, \mathrm{OH}^{+}$, and $\mathrm{SH}^{+}$. Having the most prominent line near the $\mathrm{H}_{2} \mathrm{O}$ para ground-state line at $1113.3 \mathrm{GHz}, \mathrm{H}_{2} \mathrm{O}^{+}$is serendipitously detected in many Herschel observations. $\mathrm{H}_{2} \mathrm{O}^{+}$and $\mathrm{OH}^{+}$are detected in absorption by the interstellar medium (Bruderer et al. 2010b; Gerin et al. 2010; Falgarone et al. 2010; Neufeld et al. (2010); Ossenkopf et al. 2010; Schilke et al. 2010), but also near the systemic velocity of other high-mass YSOs (Wyrowski et al. 2010b).

Several lines are found in absorption, indicated in Table 1 by negative peak values relative to the continuum. All lines predominantly in absorption $\left(\mathrm{NH}, \mathrm{OH}^{+}, \mathrm{H}_{2} \mathrm{O}^{+}\right)$originate in molecules in the ground state with a $J=1$ level energy exceeding $47 \mathrm{~K}$. Lines of $\mathrm{CH}$ and $\mathrm{SH}^{+}$, observed in emission, are transitions from 
Table 1. Frequency, upper level energy, and Einstein coefficient of molecules and lines observed by Herschel/HIFI towards W3 IRS5.

\begin{tabular}{|c|c|c|c|c|c|c|c|c|c|c|}
\hline $\begin{array}{l}\text { Mole- } \\
\text { cule }\end{array}$ & $\begin{array}{l}\text { Tran- } \\
\text { sition }\end{array}$ & $\begin{array}{r}\text { Frequency } \\
{[\mathrm{GHz}]}\end{array}$ & {$[\mathrm{K}]$} & $\begin{array}{l}A_{\mathrm{ul}} \\
{\left[\mathrm{s}^{-1}\right]}\end{array}$ & $\begin{array}{r}\text { Line peak } \\
T_{m b} \\
{[\mathrm{mK}]}\end{array}$ & $\begin{array}{r}\text { Line } \\
\text { width } \\
{\left[\mathrm{km} \mathrm{s}^{-1}\right]}\end{array}$ & $\begin{array}{r}\text { Line } \\
\text { shift } \\
{\left[\mathrm{km} \mathrm{s}^{-1}\right]}\end{array}$ & $\begin{array}{r}\text { Line flux } \\
\int T_{\mathrm{mb}} \mathrm{d} v \\
{\left[\mathrm{~K} \mathrm{~km} \mathrm{~s}^{-1}\right]}\end{array}$ & $\begin{array}{r}\text { Absorption } \\
\int \tau \mathrm{d} v \\
{\left[\mathrm{~km} \mathrm{~s}^{-1}\right]}\end{array}$ & $\begin{array}{r}\text { Column } \\
\text { density } \\
{\left[\mathrm{cm}^{-2}\right]}\end{array}$ \\
\hline$\overline{\mathrm{CH}}$ & $1_{-1}-1_{1}$ & $536.7611^{a}$ & 25.76 & $6.4(-4)$ & $740 \pm 7$ & 10.6 & +1.6 & $14.8 \pm 0.02$ & & $1.3(13)$ \\
\hline $\mathrm{NH}$ & $1_{1}-0_{1}$ & $999.9734^{a}$ & 47.99 & $5.2(-2)$ & $-446 \pm 13$ & 2.7 & -0.8 & & $3.20 \pm 0.02$ & $4.0(13)$ \\
\hline SH & $3_{1}-2_{-1}$ & $1447.0123^{a}$ & 640.6 & $8.1(-3)$ & $<220$ & & & $<0.98$ & & $<5.0(11)$ \\
\hline $\mathrm{OH}^{+}$ & $1_{1}-0_{1}$ & $1033.1186^{a}$ & 49.58 & $1.8(-2)$ & $-790 \pm 21$ & 5.9 & +34.8 & & $14.6 \pm 0.03$ & 7.1(13) \\
\hline $\mathrm{OH}^{+}$ & $2{ }_{1}-1_{1}$ & $1892.2271^{a}$ & 140.4 & $5.9(-2)$ & $<255$ & & & $<1.13$ & & $<1.1(11)$ \\
\hline $\mathrm{NH}^{+}$ & $1_{\frac{3}{2}-}-1_{\frac{1}{2}+}$ & $1019.2107^{d}$ & 48.91 & $5.5(-2)$ & $<0.032$ & & & $<0.14$ & & $<5.2(9)$ \\
\hline $\mathrm{SH}^{+}$ & $1_{2 \frac{5}{2}}^{2}-0_{1 \frac{3}{2}}^{2}$ & $526.0479^{a}$ & 25.25 & $9.7(-4)$ & $65 \pm 3$ & 4.4 & -0.3 & $0.73 \pm 0.1$ & & $4.1(11)$ \\
\hline $\mathrm{SH}^{+}$ & $2_{3 \frac{7}{2}}-1_{2 \frac{5}{2}}$ & $1082.9117^{a}$ & 77.2 & $9.1(-2)$ & $<37$ & & & $<0.17$ & & $<3.9(10)$ \\
\hline $\mathrm{SH}^{+}$ & $3_{4 \frac{9}{2}}-2_{3 \frac{7}{2}}$ & $1632.5179^{a}$ & 155.6 & $3.1(-2)$ & $<151$ & & & $<0.68$ & & $<9.8(10)$ \\
\hline $\mathrm{H}_{2} \mathrm{O}^{+}$ & $3_{12}-3_{03}$ & $999.8213^{c}$ & 223.9 & $2.3(-2)^{c}$ & $<46$ & & & $<0.20$ & & $<1.7(10)$ \\
\hline $\mathrm{H}_{2} \mathrm{O}^{+}$ & $1_{11 \frac{3}{2}}-0_{00 \frac{1}{2}}$ & $1115.2040^{b}$ & 53.52 & $3.1(-2)^{c}$ & $-285 \pm 24$ & 5.1 & +39.1 & & $2.21 \pm 0.03$ & 4.6(12) \\
\hline $\mathrm{H}_{3} \mathrm{O}^{+}$ & $4_{30}^{2}-3_{31}$ & $1031.2937^{e}$ & 232.2 & $5.1(-3)$ & $570 \pm 10$ & 6.2 & +0.5 & $3.8 \pm 0.3$ & & $9.7(11)$ \\
\hline $\mathrm{H}_{3} \mathrm{O}^{+}$ & $4_{20}-3_{21}$ & $1069.8266^{e}$ & 268.8 & $9.8(-3)$ & $230 \pm 10$ & 4.7 & -0.3 & $1.30 \pm 0.03$ & & $2.9(11)$ \\
\hline $\mathrm{H}_{3} \mathrm{O}^{+}$ & $6_{21}-6_{20}$ & $1454.5625^{e}$ & 692.6 & $7.1(-3)$ & $<245$ & & & $<0.66$ & & $<3.8(11)$ \\
\hline $\mathrm{H}_{3} \mathrm{O}^{+}$ & $2_{11}-2_{10}$ & $1632.0910^{e}$ & 143.1 & $1.7(-2)$ & $145 \pm 46$ & 6.7 & +0.3 & $1.20 \pm 0.12$ & & $3.7(11)$ \\
\hline
\end{tabular}

Notes. The numbers in parentheses give the decimal power. Negative peak fluxes signify background minus line temperature of lines in absorption. Line widths refer to the FWHP value of the most intense line peak or absorption and its line shift to the systemic velocity of $-38.4 \mathrm{~km} \mathrm{~s}^{-1}$. Nondetected peak fluxes are $5 \sigma$ upper limits at $1 \mathrm{~km} \mathrm{~s}^{-1}$ resolution, and non-detected line fluxes assume a $5 \mathrm{~km} \mathrm{~s}^{-1}$ line width. The column densities refer to the upper (emission) or lower (absorption) state. Molecular data are taken from: ${ }^{(a)}$ CDMS (Müller et al. 2001), ${ }^{(b)}$ Mürtz et al. (1998), ${ }^{(c)}$ Bruderer (2006), ${ }^{(d)}$ Hübers et al. (2009), ${ }^{(e)}$ JPL catalogue.

a $J=1$ level of energy less than $26 \mathrm{~K}$. We do not detect $\mathrm{SH}$ and $\mathrm{NH}^{+}$above the $5 \sigma$ limit.

The observed line shifts are within $\pm 2 \mathrm{~km} \mathrm{~s}^{-1}$ of the YSO, thus within the accuracy of the frequency calibration, spectral resolution, and molecular data. The only deviations in Table 1 are $\mathrm{OH}^{+}$and $\mathrm{H}_{2} \mathrm{O}^{+}$. Their strongest peaks, both in absorption, are shifted by $34-39 \mathrm{~km} \mathrm{~s}^{-1}$ relative to the YSO, similar to an absorption feature in [C II].

Figure 2 illustrates a possible explanation of the exceptional line shifts of $\mathrm{OH}^{+}$and $\mathrm{H}_{2} \mathrm{O}^{+}$. In both cases, a theoretically strong hyperfine structure line corresponds to a smaller absorption dip within less than $5 \mathrm{~km} \mathrm{~s}^{-1}$ of the YSO. For $\mathrm{OH}^{+}$, the lowfrequency line (a doublet) is blended with the high-frequency line of another, red-shifted $\mathrm{OH}^{+}$component. We thus interpret the spectra of $\mathrm{OH}^{+}$and $\mathrm{H}_{2} \mathrm{O}^{+}$as the superimposition of a component originating in the star-forming region and another component at lower frequency related to an outflow or the foreground interstellar medium near zero velocity in the LSR.

The line widths in Table 1 are between 4 and $7 \mathrm{~km} \mathrm{~s}^{-1}$, measured at the component with the systemic velocity of the YSO. Two lines attract attention: (i) $\mathrm{CH}$ has an absorption feature near the YSO velocity. This absorption reduces the peak flux and widens the line profile at half power; (ii) The line width of $\mathrm{NH}$ is extremely narrow in all hyperfine structure components.

The lines are generally symmetric. A remarkable exception is $\mathrm{OH}^{+}$, showing blue tails. We note that the shifted lines of $\mathrm{OH}^{+}$ (unblended line) and $\mathrm{H}_{2} \mathrm{O}^{+}$are 17.7 and $11.1 \mathrm{~km} \mathrm{~s}^{-1}$ wide, respectively, indicating that they have a different origin from the component at zero systemic velocity.

Several lines in Fig. 1 show slightly blue-shifted absorption and red-shifted emission, thus a P-Cyg-like behavior. Figure 2 compares them with the $[\mathrm{C} \mathrm{II}]$ line. $\mathrm{CH}$ (strongest component) exhibits similar absorption to [C II], their peak absorptions being at $-1.6 \mathrm{~km} \mathrm{~s}^{-1}$ and $-1.7 \mathrm{~km} \mathrm{~s}^{-1}$, respectively, relative to the YSO motion. $\mathrm{OH}^{+}$and $\mathrm{H}_{2} \mathrm{O}^{+}$differ, having absorption peaks at -2.5 and $-4.8 \mathrm{~km} \mathrm{~s}^{-1}$, respectively, and emission peaks at 4.2 and $3.5 \mathrm{~km} \mathrm{~s}^{-1}$.

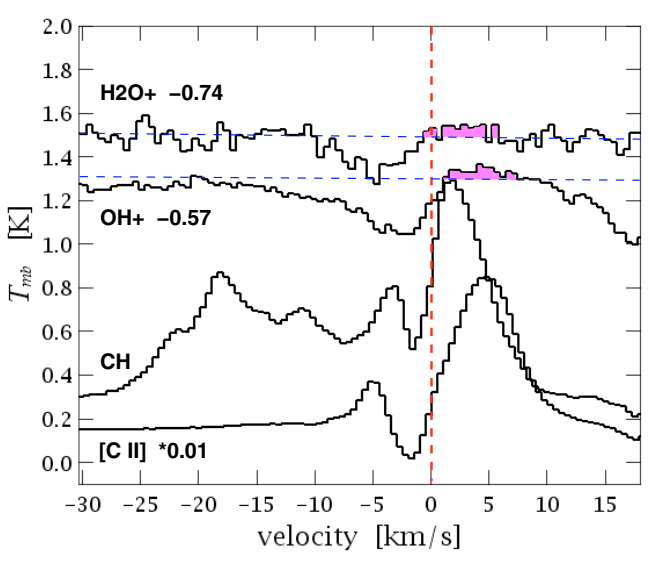

Fig. 2. Light hydrides with P Cygni type absorption features compared with [C II]. The flux scales are reduced and shifted for better visibility. The velocity is relative to the systemic velocity of W3 IRS5, shown by a vertical dashed line. The horizontal dashed lines indicate the continuum level.

Column densities are given in Table 1 for the upper energy level of transitions in emission, and for the lower energy level of lines in absorption. The fine/hyperfine structure lines were summed over all components using their statistical weight (see Bruderer et al. 2010b, for details). The observed parameters of $\mathrm{H}_{3} \mathrm{O}^{+}$at $1031 \mathrm{GHz}$ and $1069 \mathrm{GHz}$ are extracted from blends (see Fig. 1) by fitting Gaussians.

A rotational diagram can be constructed for $\mathrm{H}_{3} \mathrm{O}^{+}$(see Appendix). The data can be fitted with a straight line, suggesting that (i) the ortho-to-para ratio is compatible with unity; and (ii) a unique rotational temperature of $239 \mathrm{~K}$ (error range 205-285 K) exists. It indicates that the $\mathrm{H}_{3} \mathrm{O}^{+}$emission originates, on weighted average, from regions with equal or even hotter kinetic temperature, or, if pumped, radiation temperature. 


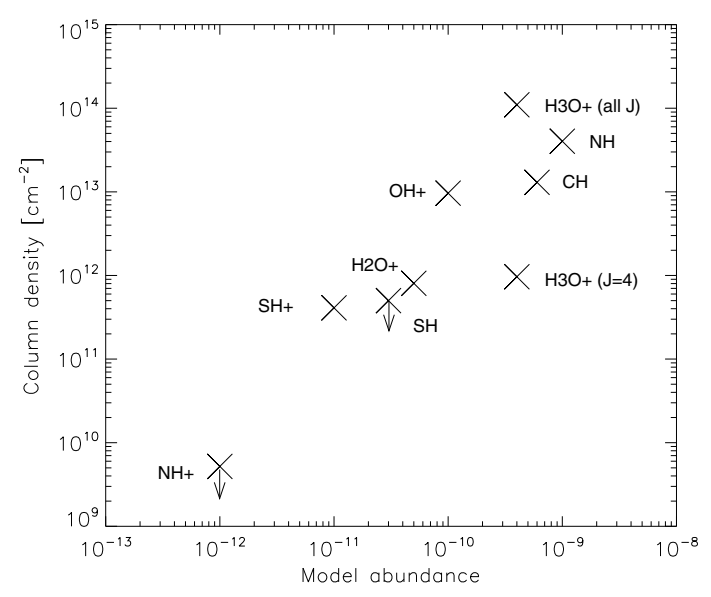

Fig. 3. Comparison of low- $J$ level column density with predicted beamaveraged abundance relative to $\mathrm{H}_{2}$ from a $2 \mathrm{D}$ model by Bruderer et al. (2010a).

\section{Discussion and conclusions}

The observed shifts of the line peaks relative to the systemic velocity of the YSO are small and suggest that the lines originate in the star-forming region, not in the foreground interstellar medium. This may not be the case, however, for the components $\mathrm{OH}^{+}$and $\mathrm{H}_{2} \mathrm{O}^{+}$shifted by a larger amount to roughly to the velocity $V_{\mathrm{LSR}}=0$.

Additional evidence of the origin comes from the lines in emission, which are produced at critical densities (if known) of order $>10^{7} \mathrm{~cm}^{-3}$. Even lines predominantly in absorption, such as $\mathrm{OH}^{+}$and possibly $\mathrm{H}_{2} \mathrm{O}^{+}$, have a small emission feature in the red wing, thus a $\mathrm{P}-\mathrm{Cyg}-\mathrm{like}$ profile, near the systemic velocity. This is the first report of Galactic $\mathrm{OH}^{+}$and $\mathrm{H}_{2} \mathrm{O}^{+}$in emission. Figure 2 shows the similarity of the profiles of $\mathrm{OH}^{+}$and $\mathrm{H}_{2} \mathrm{O}^{+}$. The absorption in $\mathrm{CH}$ may be caused by self-absorption, but is similar to that in [C II], which is found in emission at the off position. Absorption lines in star-forming regions need detailed modeling and radiation transfer calculations beyond the scope of this letter.

The ground-state column densities of the component moving with the YSO are $8.1 \times 10^{11} \mathrm{~cm}^{-2}$ for $\mathrm{H}_{2} \mathrm{O}^{+}$, and $9.7 \times 10^{12} \mathrm{~cm}^{-2}$ for $\mathrm{OH}^{+}$. The values for the red-shifted components are $3.8 \times$ $10^{12} \mathrm{~cm}^{-2}$ and $6.1 \times 10^{13} \mathrm{~cm}^{-2}$, respectively. Both components yield larger $\mathrm{OH}^{+} / \mathrm{H}_{2} \mathrm{O}^{+}$ratios than the other observations reported in this special feature.

The measured line widths are generally small $\left(<7 \mathrm{~km} \mathrm{~s}^{-1}\right)$ and show no anisotropies. We thus find no evidence of shocks except possibly in $\mathrm{OH}^{+}$, which needs to be studied in combination with shock tracers.

In Fig. 3, column densities are displayed, derived from integrated line fluxes neglecting re-emission or reabsorption of the final state. The derivation is based on Table 1, except for $\mathrm{OH}^{+}$ and $\mathrm{H}_{2} \mathrm{O}^{+}$where only the unshifted component attributed to the YSO is used. For $\mathrm{H}_{3} \mathrm{O}^{+}$, the value of the $J=4$ level as well as the one from the rotational diagram, integrated over all levels, are shown. The column densities are compared with abundances predicted by Bruderer et al. (2010a) in a two-dimensional "standard" YSO model used here as a template, assuming UV and $\mathrm{X}$-ray irradiation by a central high-mass YSO. It enhances the abundance of diatomic hydrides in the outflow walls by many orders of magnitude, such that the beam-averaged abundance is significantly changed. It is averaged over a radius of $20000 \mathrm{AU}$, or $10.9^{\prime \prime}$ at the distance of W3 IRS5.

The similarity of observations and model abundances in Fig. 3 support the scenario of hydride enrichment in outflow walls heated and irradiated by protostellar far UV.

Acknowledgements. We thank Michael Kaufman and Serena Viti for helpful comments on an early draft. This program is made possible thanks to the Swiss Herschel guaranteed time program. HIFI has been designed and built by a consortium of institutes and university departments from across Europe, Canada and the United States under the leadership of SRON Netherlands Institute for Space Research Groningen, The Netherlands and with major contributions from Germany, France, and the US. Consortium members are: Canada:CSA, U. Waterloo; France: CESR, LAB, LERMA, IRAM; Germany: KOSMA,MPIfR, MPS; Ireland: NUI Maynooth; Italy: ASI, IFSI-INAF, Osservatorio Astrofisico di Arcetri-INAF; Netherlands: SRON, TUD; Poland: CAMK, CBK; Spain: Observatorio Astronomico Nacional (IGN), Centro de Astrobiologia (CSICINT); Sweden: Chalmers University of Technology, Onsala Space Observatory, Swedish National Space Board, Stockholm University; Switzerland: ETH Zurich, FHNW; USA: Caltech, JPL, NHSC. The work on star formation at ETH Zurich is partially funded by the Swiss National Science Foundation (grant no. 200020-113556).

\section{References}

Bruderer S. 2006, Master Thesis, ETH Zurich, No. 216

Bruderer, S., Benz, A. O., Doty, S. D., et al. 2009, ApJ, 700, 872

Bruderer S., Benz, A. O., Stäuber, P., \& Doty, S. D. 2010a, ApJ, in press [arXiv: 1007.3261$]$

Bruderer, S., Benz, A. O., van Dishoeck, E. F., et al. 2010b, A\&A, 521, L44 Chavarría, L. A., herpin, F., Jacq, T., et al. 2010, A\&A, 521, L37 de Graauw, Th., Helmich, F. P., Phillips, T. G., et al. 2010, A\&A, 518, L6 Doty, S. D., Schöier, F. L., \& van Dishoeck, E. F. 2004, A\&A, 418, 1021 Falgarone, E., Ossenkopf, V., Gerin, M., et al. 2010, A\&A, 518, L118 Gerin, M., de Luca, M., \& Black, J. 2010, A\&A, 518, L110

Hollenbach, D. J., \& Tielens, A. G. G. M. 1999 Rev. Mod. Phys. 71, 173 Hübers, H. W., Evenson, K. M., Hill, C., \& Brown, J. M. 2009, J. Chem. Phys. 131,034311

Imai, H., Kameya, O., Sasao, T., et al. 2000, ApJ, 538, 751

Menten, K. M., Wyrowski, F., Belloche, A., et al. 2010, A\&A, accepted

Müller, H. S. P., Thorwirth, S., Roth, D. A., \& Winnewisser, G. 2001, A\&A, 370, L49

Mürtz, P., Zink, L. R., Evenson, K. M., \& Brown, J. M. 1998, J. Chem. Phys. 109,9744

Neufeld, D. A., Goicoechea, J. R., Sonnentrucker, P., et al. 2010, A\&A, 521, L10 Ossenkopf, V., Müller, H. S. P., Lis, D. et al. 2010, A\&A, 518, L111

Phillips, T. G., van Dishoeck, E. F., \& Keene J. 1992, ApJ, 399, 533

Rodón, J. A., Beuther, H., Megeath, S. T., \& van der Tak, F. F. S. 2008, A\&A, 490, 213

Schilke, P., Comito, C., Mueller, H. S. P., et al. 2010, A\&A, 521, L11

Stäuber, P., Doty, S. D., van Dishoeck, E. F., Jørgensen, J. K., \& Benz, A. O. 2004, A\&A, 425, 577

Stäuber, P., Doty, S. D., van Dishoeck, E. F., \& Benz, A. O. 2005, A\&A, 440, 949

Stäuber, P., Benz, A. O., Jørgensen, J. K., van Dishoeck, E. F., et al. 2007, A\&A, 466, 977

Swings, P., \& Rosenfeld, L. 1937, ApJ, 86, 483

van der Tak, F. F. S., Tuthill, P. G., \& Danchi, W. C. 2005, A\&A, 431, 993

Wyrowski, F., Menten, K. M., Güsten, R., \& Belloche, A. 2010a, A\&A, 518, A26

Wyrowski, F., van der Tak, F., Herpin, F., et al. 2010b, A\&A, 521, L34 


\section{Appendix A:}

The rotational diagram in Fig. A.1 is complemented with ground-based data for the frequency range 300-400 GHz observed at the CSO with comparable beam size by Phillips et al. (1992). The non-detection at $307 \mathrm{MHz}$ is surprising, but possibly an effect of optical depth. The data in Fig. A.1, except $307 \mathrm{GHz}$, are well fitted by a single rotational temperature of $239 \mathrm{~K}$, suggesting that the observed levels are populated according to an exponential distribution. The derived temperature and column density infer an optical depth of $\tau<0.1$ for all lines except at $307 \mathrm{GHz}$. The fitted line (dashed) corresponds to a column density of $8.5( \pm 2) \times 10^{13} \mathrm{~cm}^{-2}$, consistent with the value derived by Phillips et al. (1992). This leads to a beam averaged $\mathrm{H}_{3} \mathrm{O}^{+}$abundance of $4.2( \pm 1) \times 10^{-10}$ relative to $H$, to be compared with the theoretical value of $4 \times 10^{-10}$ reported by Bruderer et al. (2010a).

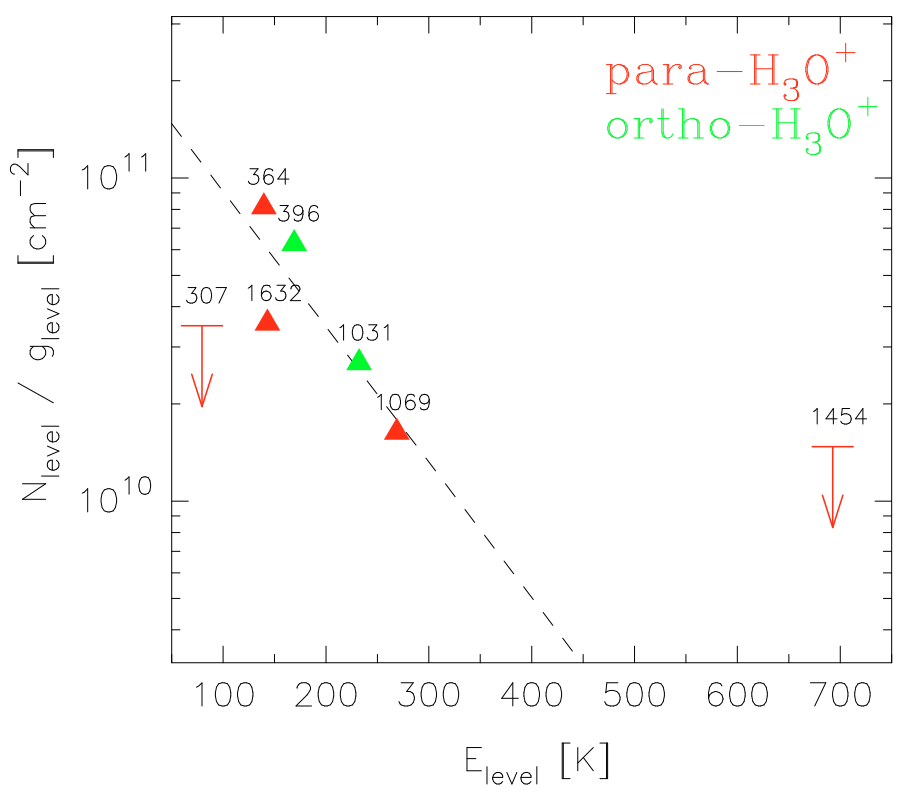

Fig. A.1. Rotational diagram of $\mathrm{H}_{3} \mathrm{O}^{+}$. Numbers indicate the frequencies in $\mathrm{GHz}$ of the observed lines.

1 Institute of Astronomy, ETH Zurich, 8093 Zurich, Switzerland e-mail: benz@astro.phys.ethz.ch

2 Leiden Observatory, Leiden University, PO Box 9513, 2300 RA Leiden, The Netherlands

${ }^{3}$ Max Planck Institut für extraterrestrische Physik, Giessenbachstrasse 1, 85748 Garching, Germany

4 School of Physics and Astronomy, University of Leeds, Leeds LS2 9JT, UK

5 INAF - Osservatorio Astrofisico di Arcetri, Largo E. Fermi 5, 50125 Firenze, Italy

${ }^{6}$ Université de Bordeaux, Laboratoire d'Astrophysique de Bordeaux, France; CNRS/INSU, UMR 5804, Floirac, France

7 National Research Council Canada, Herzberg Institute of Astrophysics, 5071 West Saanich Road, Victoria, BC V9E 2E7, Canada
8 Department of Physics and Astronomy, University of Victoria, Victoria, BC V8P 1A1, Canada

9 Department of Radio and Space Science, Chalmers University of Technology, Onsala Space Observatory, 43992 Onsala, Sweden

10 SRON Netherlands Institute for Space Research, PO Box 800, 9700 AV, Groningen, The Netherlands

11 Kapteyn Astronomical Institute, University of Groningen, PO Box 800, 9700 AV, Groningen, The Netherlands

12 Observatorio Astronómico Nacional (IGN), Calle Alfonso XII 3, 28014 Madrid, Spain

13 INAF - Istituto di Fisica dello Spazio Interplanetario, Area di Ricerca di Tor Vergata, via Fosso del Cavaliere 100, 00133 Roma, Italy

14 Department of Astronomy, The University of Michigan, 500 Church Street, Ann Arbor, MI 48109-1042, USA

15 California Institute of Technology, Division of Geological and Planetary Sciences, MS 150-21, Pasadena, CA 91125, USA

16 Centro de Astrobiología. Departamento de Astrofísica. CSIC-INTA. Carretera de Ajalvir, Km 4, Torrejón de Ardoz, 28850 Madrid, Spain.

17 Astronomical Institute Anton Pannekoek, University of Amsterdam, Kruislaan 403, 1098 SJ Amsterdam, The Netherlands

18 Department of Astrophysics/IMAPP, Radboud University Nijmegen, PO Box 9010, 6500 GL Nijmegen, The Netherlands

19 Department of Physics and Astronomy, Denison University, Granville, OH, 43023, USA

${ }^{20}$ LERMA and UMR 8112 du CNRS, Observatoire de Paris, 61 Av. de l'Observatoire, 75014 Paris, France

21 University of Waterloo, Department of Physics and Astronomy, Waterloo, Ontario, Canada

22 Observatorio Astronómico Nacional, Apartado 112, 28803 Alcalá de Henares, Spain

23 INAF - Osservatorio Astronomico di Roma, 00040 Monte Porzio catone, Italy

${ }^{24}$ Centre for Star and Planet Formation, Natural History Museum of Denmark, University of Copenhagen, Øster Voldgade 5-7, 1350 Copenhagen K., Denmark

25 Department of Astronomy, Stockholm University, AlbaNova, 10691 Stockholm, Sweden

26 California Institute of Technology, Cahill Center for Astronomy and Astrophysics, MS 301-17, Pasadena, CA 91125, USA

27 The University of Western Ontario, Department of Physics and Astronomy, London, Ontario, N6A 3K7, Canada

28 Harvard-Smithsonian Center for Astrophysics, 60 Garden Street, MS 42, Cambridge, MA 02138, USA

29 Department of Physics and Astronomy, Johns Hopkins University, 3400 North Charles Street, Baltimore, MD 21218, USA

30 Max-Planck-Institut für Radioastronomie, Auf dem Hügel 69, 53121 Bonn, Germany

31 Jet Propulsion Laboratory, California Institute of Technology, Pasadena, CA 91109, USA

32 Department of Physics and Astronomy, University of Calgary, Calgary, T2N 1N4, AB, Canada

33 Instituto de Radioastronomía Milimétrica (IRAM), Avenida Divina Pastora 7, Núcleo Central, 18012 Granada, Spain

34 KOSMA, I. Physik. Institut, Universität zu Köln, Zülpicher Str. 77, 50937 Köln, Germany

35 Institute of 4D Technologies, University of Applied Sciences NW, 5210 Windisch, Switzerland

36 Laboratory for Electromagnetic Fields and Microwave Electronics, ETH Zurich, 8092 Zurich, Switzerland 